\title{
698.
}

\section{ON A THEOREM RELATING TO COVARIANTS.}

[From the Journal für die reine und angewandte Mathematik (Crelle), t. LxxxvII. (1878), pp. 82, 83.]

The theorem given by Prof. Sylvester, Crelle, vol. Lxxxv., p. 109, may be stated as follows: If for a binary quantic of the order $i$ in the variables, we consider the whole system of covariants of the degree $j$ in the coefficients, then

$$
\Sigma \theta(k+1)=\frac{\Pi(i+j)}{\Pi(i) \Pi(j)},
$$

where $\theta$ denotes the number of asyzygetic covariants of the order $\theta$ in the variables, the values of $\theta$ being $\ddot{j}, \ddot{j}-2, \ddot{j}-4, \ldots, 1$ or 0 , according as $\ddot{i j}$ is odd or even.

In the case of the binary quintic $(a, \ldots \chi x, y)^{5},(i=5)$, we have a series of verifications in the Table 88 of my "Ninth Memoir on Quantics," Phil. Trans. vol. cLxI. (1871), [462]: viz. writing the small letters $a, b, c, \ldots, u, v, w$ (instead of the capitals $A, B$, etc.) to denote the covariants of the quintic, $a$, the quintic itself, degree 1 , order 5 , or as I express it, deg-order 1.5: b, the covariant deg-order 2.2, etc., the whole series of deg-orders being

$$
\begin{aligned}
& a, \quad b, \quad c, \quad d, \quad e, \quad f, \quad g, \quad h, \quad i, \quad j, \quad k, \quad l \text {, } \\
& \text { 1.5, 2.2, 2.6, 3.3, 3.5, 3.9, 4.0, 4.4, 4.6, 5.1, 5.3, 5.7, } \\
& m, \quad n, \quad o, \quad p, \quad q, \quad r, \quad s, \quad t, \quad u, \quad v, \quad w \text {, } \\
& 6.2, \quad 6.4,7.1,7.5, \quad 8.0,8.2,9.3,11.1, \quad 12.0, \quad 13.1,18.0 \text {, }
\end{aligned}
$$

then the table shows for each deg-order, the several covariants of that deg-order, and 
the number of them which are asyzygetic; for instance, $i=5$ as above, $j=6$, an extract from the table is

\begin{tabular}{|c|c|c|c|c|}
\hline$j$ & $k$ & $\theta$ & & $(k+1) \theta$ \\
\hline \multirow[t]{16}{*}{6} & 30 & 1 & $a^{6}$ & 31 \\
\hline & 28 & 0 & & 0 \\
\hline & 26 & 1 & $a^{4} c$ & 27 \\
\hline & 24 & 1 & $a^{3} f$ & 25 \\
\hline & 22 & 2 & $a^{4} b, a^{2} c^{2}$ & 46 \\
\hline & 20 & 2 & $a^{3} e, a c f$ & 42 \\
\hline & 18 & 3 & $a^{3} d, a^{2} b c, c^{3}, f^{2}$ & 57 \\
\hline & 16 & 2 & $a^{2} i, a b f, a c e$ & 34 \\
\hline & 14 & 4 & $a^{2} b^{2}, a^{2} h, a c d, b c^{2}, e f$ & 60 \\
\hline & 12 & 3 & $a b e, a l, c e, d f$ & 39 \\
\hline & 10 & 4 & $a^{2} g, a b d, b^{2} c, c h, e^{2}$ & 44 \\
\hline & 8 & 2 & $a k, b i, d e$ & 18 \\
\hline & 6 & 4 & $a j, b^{3}, b h, c g, d^{2}$ & 28 \\
\hline & 4 & 1 & $n$ & 5 \\
\hline & 2 & 2 & $b g, m$ & 6 \\
\hline & 0 & 0 & & 0 \\
\hline
\end{tabular}

where, for instance deg-order 6.14 , the covariants are $a^{2} b^{2}, a^{2} h$, acd, $b c^{2}, e f$, but the number against these in the third column being (not 5 but) 4 , the meaning is that there exists between these five terms one syzygy, making the number of asyzygetic covariants of the deg-order 6.14 to be 4 . The second column thus in fact contains the several values of $k$, and the third column the corresponding values of $\theta$; whence, forming the several products $(k+1)$ as shown, the sum of these is as it should be $=462$.

Cambridge, 13 July, 1878. 